\title{
A Survey of State-of-the-Art Energy Efficiency Routing Protocols for MANET
}

\author{
https://doi.org/10.3991/ijim.v14i09.13939 \\ Vu Khanh Quy $\left({ }^{\bowtie}\right)$, Vi Hoai Nam and Dao Manh Linh \\ Hung Yen University of Technology and Education, Hung Yen, Vietnam \\ quyvkeutehy.edu.vn
}

\begin{abstract}
In recent years, the Mobile Ad Hoc Network (MANET) has a vital role in many fields such as healthcare, military, rescue and smart city. Because of its mobility and ad hoc, each mobile network node acts as a router with limited battery energy. The out of energy network node not only affects its own ability to transfer/receive packets but also affects the overall performance of the entire network. Therefore, an energy-efficient routing protocol will optimise the efficiency of MANET. Many routing protocols have been proposing to solve this problem. In this paper, we surveyed the proposed energy-efficient routing protocols in the last decade for MANET. Based on the survey results, we propose open research directions. We hope that this work will make a strong motivation in the field of energy-efficient routing research for MANET. The survey results are also a vital basis to serve the in-depth researches in the routing field for MANET of our research team.
\end{abstract}

Keywords-Routing Protocols, Mobile Ad hoc Network, Energy Efficiency, MANET

\section{Introduction}

With the vigorous development of mobile communication systems, according to expect of Cisco VNI (Visual Network Index), by 2023, over 29.3 billion global devices will be networked, up from 18.4 billion in 2018. Moreover, there will have over 13 billion mobile devices connected to the network, up from 8.8 billion in 2018. Its also expect that each capita will have 1.6 mobile devices connected. Especially mobile devices equipped with M-to-M modules are the basis for forming Mobile Ad hoc Network (MANET) [1]. These devices will consume enormous energy. Therefore, energy efficiency becomes a vital standard for the all communication system. A next-generation network just introduced is the $5 \mathrm{G}$ network. Its energy efficiency has been defined as one of the main requirements targeting 100 fold improvement over the $4 \mathrm{G}$ systems [23].

Due to its mobility and ad hoc, each mobile node act as a router for receiving and forwarding packets with the limited battery energy level. When an out of energy node not only affects its own ability to receive/transmit packets but also affects the overall performance of the network. In a MANET, since network nodes must cooperate to 
transmit packets, routing protocols are particularly crucial in saving energy [3-5]. The traditional routing protocols used for MANET such as AODV (Ad-hoc On-demand Distance Vector) [24] or DSR (Dynamic Source Routing) [25] using hop count routing metric are ineffective [4-5].

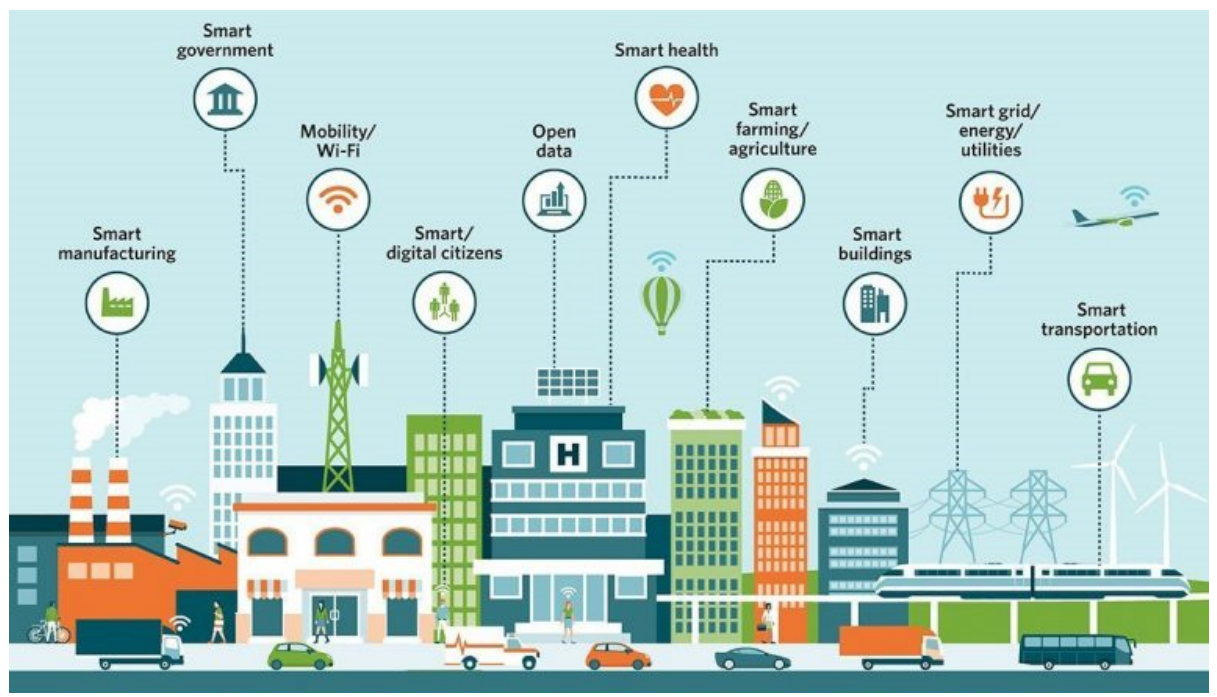

Fig. 1. An illustration of MANET application as a communication platform in smart cities

In this study, we survey routing protocols for MANET approached towards energy efficiency over the last decade published in the IEEE Explore Digital Library. The routing protocols in this survey are arranged based on two different approaches: Minimum the total energy consumption and Maximum Network Lifetime. Based on the surveyed results, we proposals many open research directions in the Discussion section. In the next section, we analysis and discussion to the surveyed protocols.

\section{Review of Energy Efficiency Routing Protocols}

Because of the characteristic of MANET, mobile network nodes use batteries to store energy. Therefore, saving-energy is a particularly important issue. Minimum the energy consumed per packet is the most basic approach of saving-energy routing protocols [4, 6-9]. First, we analyse the fundamental issues of energy consumption in MANET. The energy of a network node is consumed by two primary factors, such as:

- The process of transmitting/receiving packets.

- Energy consumed by listening on from the radio environment (overhearing).

The survey results show that many saving energy routing protocols have been proposed [10-23], focusing on the following two approaches: 
- Minimum the total energy consumption (Power Control)

- Maximum Network Lifetime (Network Lifetime). Network lifetime is calculated from the time the network starts operation until the first node runs out of power; in other words, it is not enough power to send or receive packets.

Table 1. Saving Energy Routing Protocols for MANET in IEEE Digital Library, 2010 - 2019

\begin{tabular}{|c|c|c|c|c|c|c|c|}
\hline Protocol & Year & Metrics routing & Compare with & Energy & Delay & PDR & Overh. \\
\hline $\begin{array}{l}\text { AEADMRA } \\
{[10]}\end{array}$ & 2010 & Residual Energy & $\begin{array}{l}\text { ADRA, AOMDV, DSR, } \\
\text { EC-GRID }\end{array}$ & Yes & Yes & Yes & Yes \\
\hline LAER [11] & 2012 & $\begin{array}{l}\text { Link State, Drain } \\
\text { Rate }\end{array}$ & $\begin{array}{l}\text { GPSR, E-GPSR, PERRA, } \\
\text { LAER }\end{array}$ & Yes & No & Yes & Yes \\
\hline PDTMRP [12] & 2012 & Hop Count, Power & $\begin{array}{l}\text { MAODV, RMAODV, Par- } \\
\text { allel MNTMR }\end{array}$ & Yes & Yes & Yes & Yes \\
\hline LRPH [13] & 2013 & Power & LRPS, MC, DSR & Yes & Yes & Yes & Yes \\
\hline ERBA [14] & 2013 & Link State & AODV, ROMSGP & Yes & Yes & Yes & No \\
\hline EPRDSR [15] & 2014 & Power & DSR, MPTR & Yes & Yes & Yes & No \\
\hline $\begin{array}{l}\text { DEL-CMAC } \\
{[16]}\end{array}$ & 2015 & $\begin{array}{l}\text { Location, Power Al- } \\
\text { location }\end{array}$ & $\begin{array}{l}\text { IEEE } 802.11 \text { DCF, Coop- } \\
\text { MAC }\end{array}$ & Yes & Yes & No & No \\
\hline $\begin{array}{l}\text { FF-AOMDV } \\
{[18]}\end{array}$ & 2017 & $\begin{array}{l}\text { Hop count, Residual } \\
\text { Energy, Location }\end{array}$ & AOMDV, AOMR-LM & Yes & Yes & Yes & Yes \\
\hline OEFS [19] & 2017 & Residual Energy & E-CHANET & Yes & Yes & Yes & No \\
\hline CRCPR [20] & 2018 & $\begin{array}{l}\text { Link State,Residual } \\
\text { Energy, }\end{array}$ & DEHAR, AODV-EHA & Yes & Yes & Yes & No \\
\hline SynFAnt [21] & 2018 & $\begin{array}{l}\text { Bandwidth, Life- } \\
\text { time, Reliability }\end{array}$ & $\begin{array}{l}\text { EFMMRP, ETX,LOADng, } \\
\text { EELB-Mega }\end{array}$ & Yes & Yes & Yes & No \\
\hline $\begin{array}{l}\text { MEQSA- } \\
\text { OLSRv2 [22] }\end{array}$ & 2018 & Residual Energy & $\begin{array}{l}\text { Several OLSR based proto- } \\
\text { cols }\end{array}$ & Yes & Yes & Yes & Yes \\
\hline $\begin{array}{l}\text { EIMO- } \\
\text { ESOLSR [23] }\end{array}$ & 2019 & $\begin{array}{l}\text { Bandwidth, Resid- } \\
\text { ual Energy }\end{array}$ & Tradition Protocols & Yes & Yes & Yes & Yes \\
\hline
\end{tabular}

\subsection{Power control direction}

The goal of this strategy is to minimise overall energy consumption. Approaching in that direction, one of the first protocols proposed by Singh et al. [8] was MTPR (Minimal Total Power Routing). Let $e_{l}$ is the energy portion consumed to transmit or receive a packet on the link $l$. So, the total energy consumed to transmit a packet from the source node $\mathrm{S}$ to the destination node $\mathrm{D}$ on the route $\mathrm{p}$ can be determined as follows:

$$
E_{p}=\sum_{l \in p} e_{l}
$$

This routing metric will prioritise route that has many shorter hops than the route has fewer hop numbers, but the distance of each hop is longer. Although this solution is more efficient energy and less interference, it will cause bottlenecks due to traffic concentrated going to low energy cost routes.

According to this research direction, in this recent time, many studies are proposed, in [12], Wang et al. (2012) proposed a routing protocol, called PDTMRP (Power-aware Dual-Tree-based Multicast Routing Protocol) for MANETs. This focus of research is the load-balance is used to improve the lifetime of the network. To solve this problem, 
all nodes are randomly classified into two types, group- 0 and group- 1 . To achieve the load balance, two multicast trees (tree- 0 for group- 0 and tree- 1 for group- 1 ) are constructed. Results show that the packet delivery ratio and the time delay of the proposed protocol outperform that of MAODV, RMAODV and Parallel MNTMR protocols. Moreover, the traffic load can be balanced, and the network lifetime can be prolonged.

In [13], Peng Zhao et al. (2013) proposed a routing protocol, called LRPH (Loosevirtual-clustering-based (LVC) Routing Protocol for power heterogeneous MANETs. This focus of research is to reduce the interference raised by high-power nodes, by the way, avoid packet forwarding via high-power nodes. Via the combination of analytical modelling, simulations, and real-world experiments, authors show that the effectiveness of LRPH on improving the performance of power heterogeneous MANETs.

In [15], Shiva Shankar et al. (2014) proposed a routing protocol, called EPRDSR (Efficient Power Routing DSR) purpose improve performance overall network, extend the lifetime of each node and prolong the lifetime of each connection. This focus of research proposes an efficient power scheme in the whole MANET. In the route discovery phase, the EPRDSR constraints the bandwidth and power metrics into the route select schema. The simulation results have indicated that the performance of the EPRDSR protocol increases the network lifetime by $60-65 \%$ as compared with the DSR and the MTPR protocols.

In [16], Wang et al. (2015) proposed a routing protocol, namely DEL-CMAC (Distributed Energy-adaptive Location-based CMAC protocol) for MANET, with the objective is to improve the performance of the MANETs in terms of network lifetime and energy efficiency. The focus of research takes the energy consumption into account and propose a relay selection strategy to choose the best relay terminal and a cross-layer optimal power allocation scheme to set the transmitting power. The simulation result shows that the DEL-CMAC significantly prolongs the network lifetime compares with the IEEE 802.11 DCF and CoopMAC under various circumstances.

\subsection{Network lifetime direction}

The goal of this strategy is to extend the maximum lifetime of all nodes throughout the network. The solution is to balance the energy consumption of all nodes in the network. One of the first routing protocols proposed to follow this direction is Chao et al. (2003) [17]. The focus of this proposal is to use remaining battery capacity as the routing metric instead of the traditional hop number. The ratio of the remaining battery capacity of a node, $R_{b r c}$ is determined, as follows:

$$
R_{\text {brc }}=\frac{E_{i}}{E_{\max }}=\frac{\text { Battery remaining capacity }}{\text { Battery full capacity }}
$$

Also in this direction, to improve efficient energy, C.-K. Toh (2001) [9] proposed a routing metric, called MBCR (Minimum Battery Cost Routing), which purpose to selected the route has richer energy. The authors define $c_{i}^{t}$, is the battery capacity of a node $n_{i}$ at time $t$. The battery cost function of node $n_{i}$ can be determined, as follows:

$$
f_{i}\left(c_{i}^{t}\right)=1 / c_{i}^{t}
$$


As the battery capacity decreases, the value of cost function for the node $n_{i}$ will increases. Let $R_{j}$ is the total cost on the route $\mathrm{j}$ has $\mathrm{D}$ nodes, authors obtained:

$$
R_{j}=\sum_{i=0}^{D_{j}-1} f_{i}\left(c_{i}^{t}\right)
$$

Therefore, to find the route with the maximum remaining battery capacity, authors select the route $i$ that has the minimum battery cost, $R_{i}=\min \left\{R_{j} \mid \mathrm{j} \in \mathrm{A}\right\}$, where A is the set containing all possible routes. By directly incorporate the battery capacity in the routing protocol, MBCR always finds the route with the maximum remaining battery capacity. However, because only consider the total remaining battery capacity, the selected route still can contain the exhausted node. To solve this problem, the authors proposed MMBCR (Minimal Maximum Battery Cost Routing) metric for purpose excludes the exhausted node in the selected route. The objective function (Eq. 4) is modified, as follows:

$$
R_{i}=\max _{i \in \text { route }_{j}} f_{i}\left(c_{i}^{t}\right)
$$

Where, like the MBCR metric, the route $i$ obtained, as follows:

$$
R_{i}=\min \left\{R_{j} \mid j \in A\right\}
$$

This metric always tries to avoid routes containing the energy exhausted node. Therefore, the network lifetime can be improved.

Since there is no yet guarantee that the transmission power total on the route is selected is minimum. To solve this issue, in [9], C.-K. Tok (2001) proposed the CMMBCR (Conditional MMBCR) scheme. The basic idea behind CMMBCR is using a concept called Threshold to increasing the lifetime of each node and using the more efficient battery, as follows:

- If all of the nodes in the candidate routes of the source-destination pair have the remaining battery capacity is higher than the threshold value, then, the route has the minimum transmission power will be selected.

- If the candidate route contains the node wich remaining battery capacity is lower than the threshold value, then, uses the MMBCR metric to select the route.

Based on these research directions, in recent time, many works are proposed. Accordingly, Wu et al. (2010) [10] proposed an AEADMRA (Ant-based Energy-aware Disjoint Multipath Routing Algorithm) protocol for MANETs. The routing algorithm is based on swarm intelligence and especially on the ant colony-based meta-heuristic. This protocol can discover multiple energy-aware routing paths with a low routing overhead. The simulation experiments indicated that the performance and network lifetime of AEADMRA outperform other some traditional protocols.

In [11], Floriano De Rango et al. (2012) proposed a routing protocol based on the joint metric of link stability and energy drain rate, called LAER (Link-stAbility and Energy-aware Routing protocols). It bases on the local topology knowledge; use a greedy technique based on a joint metric and a modified perimeter forwarding strategy for the recovery from the local maximum. The performances of the proposed protocol 
have been compared with the other three protocols, such as GPSR, E-GPSR, and PERRA. The result simulation shows that LAER protocol inherits the scalability of GPSR and E-GPSR, improving the performance and lifetime outperforms comparison with other protocols.

With the objective is to improve the performance of the VANET, in [14], Zhang et al. (2013) proposed ERBA (Energy-Efficient Routing) protocol using movement trends. To solve this issue, the research proposed scheme employs the driving patterns, vehicle category and intersection information to build reliable and stable routes among vehicles. Experimental results over real urban scenarios extracted from ShanghaiGrid project demonstrate that ERBA outperforms the compared routing schemes in VANETs in terms of the end-end delay, the packet delivery ratio and the path duration time.

In [18], Taha et al. (2017) proposed an efficiency energy routing protocol, called FFAOMDV (AOMDV with the Fitness Function) for MANET. The focus of research is to improve the AOMDV routing protocol by applying the fitness function technique to optimise energy consumption. The fitness function is used to the optimal path from a source node to a destination node to reduce energy consumption. The simulation results on NS2 clearly show that the proposed FF-AOMDV improve outperform performance metrics such as throughput, packet delivery ratio and end-to-end delay compare AOMDV and AOMR-LM routing protocols.

In [19], Rehman et al. (2017), proposed a novel routing protocol, called OEFS (Ondemand Energy-based Forwarding Strategy). The focus of research takes the residual energy of the nodes into account during the entire communication process. The OEFS has four main characteristics follow as: Take the residual energy of the node into account in entire processing and using a forwarding mechanism based on energy. When a node in the 'Danger' status, it will focus on the delivery data and reduces the Interest and Data packets flooding in the network to mitigate packet collisions. The simulation results show that the OEFS outperforms the state-of-the-art protocol in terms of download time, the total number of interest propagation, and data redundancy in the network and enables nodes to consume less amount of energy.

In [20], Bai et al. (2018), proposed a routing protocol, called CRCPR (ConstructiveRelay-based CooPerative Routing) for MANETs. The focus of research is proposing topological information stored and maintained in the CooPerative Table and Relay Table and a new route selection mechanism which takes into account energy consumption, energy harvesting, and link break probability, to determine an appropriate route across a network. Simulation results show that CRCPR improves up to $60 \%$ network throughput and $40 \%$ prolonged network lifetime than traditional protocols.

In [21], Kacem et al. (2018) proposed a routing protocol, called SynFAnt (Synchronized Fuzzy Ant System) with the main objective is to find the least-cost investment in nominal capacities that ensures the routing of nominal traffic. The objective of the proposal is to guarantees packets survivability in any case of an arc or node failure in MANET by using a synchronised fuzzy transition approach, where the ant system is used to find a solution for the problem of uncertainty events in ad hoc networks. This proposed solution to intelligently control the flow in MANETs. It has the advantages such: preventive and quickly adapted to the changes that occur; detect faulty nodes and speedily propose new routing tables, to avoid high transmission delays that lead to 
packet losses. The simulation results show that this protocol improves the packet delivery ratio, the throughput, the end-to-end delay, and the acceptance rate of the QoS flows compared to four other protocols.

In [22], Jabbar et al. (2018) proposed a routing protocol, called MEQSA-OLSRv2 (Multipath Energy and Quality of Service Aware OLRSv2), improved from OLSRv2 protocol with the objective are saving energy and QoS guarantees for the data transmission in the MANET-WSN convergence scenarios of IoT networks. The focus of research is the proposal a method to node rank according to MCNR (Multi-Criteria Node rank Metric), which is formed base on multiple parameters such as the lifetime, residual battery energy, idle time, speed, queue. Besides, this study also proposed a method to select a multipoint relay (MPR) set of nodes by using energy and QoS-aware MPR selection mechanism for flooding topological information. Simulation results show that the performance of the MEQSA-OLSRv2 outperforms comparing with the conventional routing protocols in terms of QoS, saving energy, and decreases the energy cost per packet.

In [23], Kanagasundaram et al. (2019) proposed a routing protocol, called EIMOESOLSR (Enhanced Intellects Masses Optimiser - Energy-efficient and Secure OLSR), improved from the tradition OLSR protocol to efficiency energy and security for MANET. The focus of research proposes energy and security-aware routing model for MANET including an Enhanced Intellects-Masses Optimiser (EIMO) and, a Composite Eligibility Index (CEI) which inbuilt by multimetric as available bandwidth, queue occupancy, and lifetime. The result simulation shows that the EIMO-ESOLSR outperforms other state-of-the-art protocols in terms of the performance metrics like energy consumption, total remaining time, average network lifetime.

\section{Discussion and Future Research Directions}

In this work, we surveyed routing protocols towards energy efficiency published over the last tens year (the period 2010-2019) on the IEEE Xplore Digital Library. We arrange the studies into two sets, correspond with two main approaches direction: 1) Power control and 2) Maximum network lifetime.

The survey protocols are summarised in Table 1 . The survey results show that many efficiency energy routing protocols have been proposed over the past decade. The survey shows some exciting results, specifically as follows: 


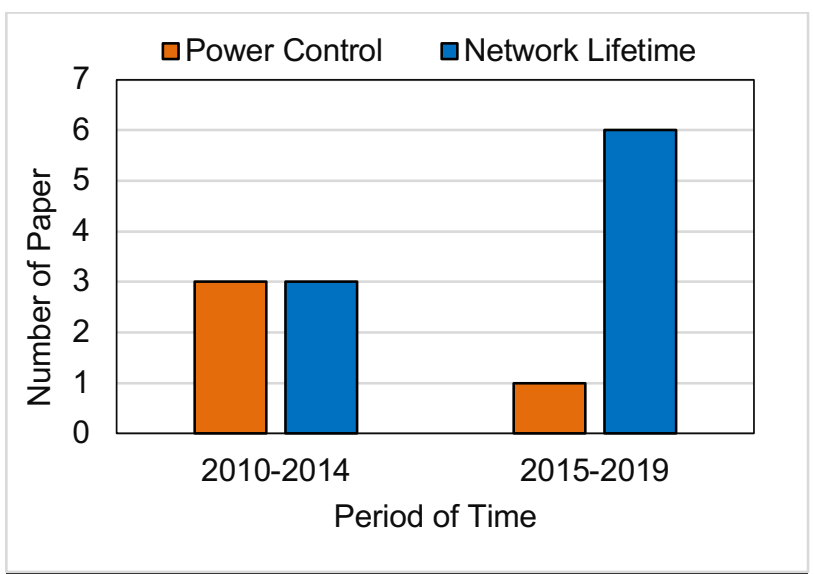

Fig. 2. Statistics of proposed protocol numbers according to periods.

The protocols number in the maximum network lifetime direction account over $70 \%$ of the total number of the proposed protocol, while the protocols in the power control direction only account for about $30 \%$, especially the recent few years, stage: 2017-2019, the protocols number in the maximum network lifetime direction huge dominate. $100 \%$ of protocols are improved from traditional known routing protocols such as AODV, DSR. The two most common routing metrics are residual energy and power, in particular, the residual energy metric is considered in over $50 \%$ of the studies. Over $90 \%$ of the studies use tradition simulation software (NS2) to evaluation performance. The typical performance criteria are network lifetime, packet delivery ratio, end-to-end delay and overhead. The network lifetime criterion is used in $100 \%$ of the studies while the two criteria: the packet delivery ratio and end-to-end delay are used in over $95 \%$ of the studies. $100 \%$ recently routing protocols in stage: $2015-2019$ use multi-metric which use metrics such as residual energy, hop count, link-state and incorporate them in a cost function to select the optimum route.

The survey results show that most of the routing protocols incorporated energy metrics (such as power or residual energy) in a cost function to ensure that the route has the lowest power consumption will be selected or to avoid exhausted nodes. Moreover, many studies combined energy information with other information such as location, hop numbers or link-state to achieve superior performance.

Survey also shows that due to the dynamic topology of MANET, no specific protocol can operate well in all situations. Each direction approach has its merits and drawbacks, as above described. The network topology and routing metrics play vital roles in deciding which protocol should be used to obtain the best performance in terms of energy efficiency. The approach base on link-state may be a good ideal for a small network. However, this approach becomes infeasible in MANET have large topology and the nodes have high mobility, due to the system need an amount of large bandwidth and traffic for send packet control as well as consumption much energy. The approach based on improvement from the dynamic source routing protocol better operation in large MANETs and high mobility due to they use less control traffic than link-state approach. 
However, due to no use a topology map, the approach may cause higher delay because the nodes must spend time in the route discovery when they need to deliver data packets.

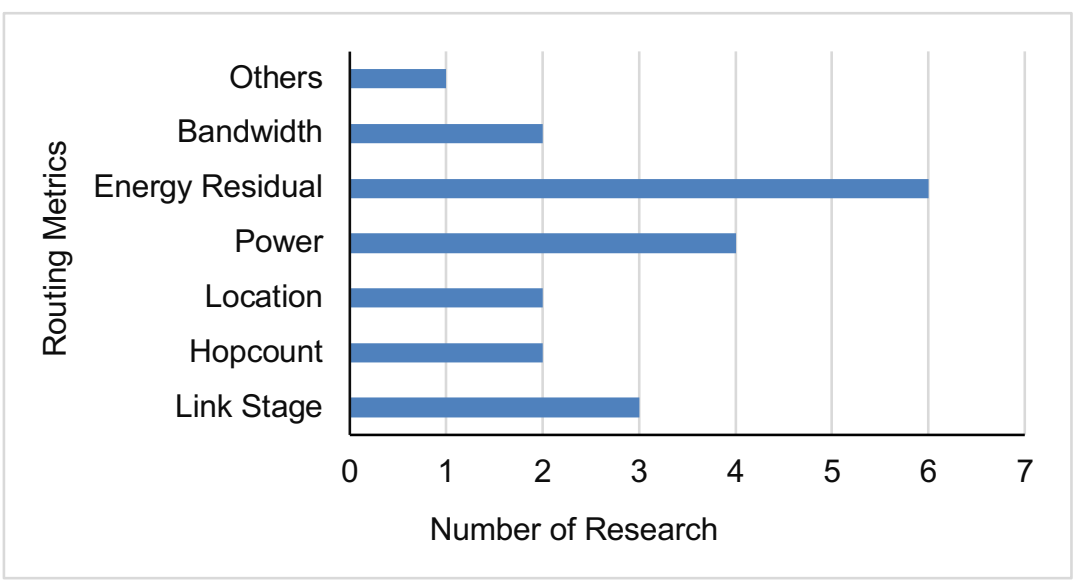

Fig. 3. Statistics of basic Routing Metrics in the Surveyed Protocols.

After the survey and analytic the routing protocols above, we see emerging many challenges and open issues in energy efficiency routing field in MANET. These issues require the development of more and more efficient and flexibly protocols. Based on these analyses, we propose some research direction in the future. We believe that, in the next future, with the booming development of the new generation networks and IoT networks, the most of devices joint in the network are smart mobile devices, use M-to$M$ communication and saving energy solutions. Therefore MANET will continue to be research topics of special interest, in particular, routing protocols to maximize network lifetime.

The survey results show that each routing metric is designed for a specific purpose [26]. Fig. 3 is a statistical chart of the routing metrics used for protocols surveyed in Table 1. The results show that common routing metrics are: Energy Residual, Hop count, Link-state, and Power. Besides, observing Table 1, we find that the recent proposed studies tend to use integrated metrics, which are combined from different metrics. In our view, in order to meet the various goals of the network, this trend is inevitable. Therefore, choosing the appropriate routing metrics to incorporate in the cost function will be an exciting study topic.

With the rapid development of multimedia applications, today, many voice call, video call applications allow operation on IoT systems with a charge or no fee. Researching and proposing routing protocols for multimedia applications operate on MANET is indispensable [27]. However, in practice, QoS guarantee protocols tend to choose the route with the highest throughput and lowest end-to-end delay, whereas saving energy protocols tend to choose routes to extend network lifetime. Therefore, the research of the trade-off [28] or clustering [29] methods are necessary. 
In order to expand the efficiency and capacity of MANET, hybrid architectures such as Cloud-assisted MANET proposed in many recent studies [30]. However, with limited battery energy and device configuration, offload routing techniques from MANET to the Cloud, and the Internet needs to be studied to forward complex calculate problems from MANET to the Cloud or the Internet. Therefore, researching routing techniques between MANET and other network architectures to optimise performance and energy efficiency for MANET also will be interesting topics.

\section{Conclusion}

Due to its limited battery energy and highly dynamic network architecture, the energy-efficient routing is a challenging issue for MANET. Survey of recently state-ofthe-art routing protocols in the direction of energy efficiency shows a general framework for approaching solutions to this problem. The results show that, in recent years, many routing protocols for MANETs in this direction have been studied and proposed. In this work, we surveyed routing protocols over the last decade published in the IEEE Xplore Digital Library Database from 2010 to 2019. We divided the protocols into two groups, corresponding with each purpose of the protocols, include: 1) Minimum the total energy consumption (Power Control) 2) Maximum Network Lifetime.

Besides the analyses, comparisons, we also discussed open issues about the routing protocols under consideration. Moreover, based on survey results, we have found changes in study trends over the last years as well as the identification of promising research directions in the future. The main purpose of this study is to provide an overview of the proposed energy-efficient routing protocols for MANET. We hope this work will be promoting the launch of new saving energy routing protocols for the MANET-WSN convergence scenarios of IoT networks.

\section{Acknowledgement}

The authors sincerely thank Ass Prof. Dr Nguyen Tien Ban and Ass Prof. Dr Nguyen Dinh Han for their constant support from starting to end of this research work.

\section{References}

[1] White paper. Cisco Visual Networking Index: Global Mobile Data Traffic Forecast, 20172023. Update 2018. [Online]. Available: https://www.cisco.com

[2] J. P. Josh Kumar, A.Kathirvel (2019). Analysis and Ideas for Improved Routing in MANET. International Journal of Interactive Mobile Technologie, 2019. 13(4): p. 164-177. https://doi.org/10.3991/ijim.v13i04.9928

[3] M. Alnabhan et al., (2017). Performance Evaluation of Unicast Routing Protocols in MANETs. International Journal of Interactive Mobile Technologies, 2017. 11(1): p. 84-97. https://doi.org/10.3991/ijim.v11i1.6295

[4] M. Masoudi et al., (2019) "Green Mobile Networks for 5G and Beyond", IEEE Access, vol. 7, pp. 107270-107299. https://doi.org/10.1109/ACCESS.2019.2932777 
[5] Quy V.K., Ban N.T., Nam V.H., Tuan D.M., Han N.D. (2019) Survey of Recent Routing Metrics and Protocols for Mobile Ad-Hoc Networks. In Journal of Communications, 14(2): pp. 110-120. https://doi.org/10.12720/jem.14.2.110-120

[6] Haider Th. Salim ALRikabi et al. (2020) The Application of Wireless Communication in IOT for Saving Electrical Energy. in International Journal of Interactive Mobile Technologies, 14(1): pp. 152-160. https://doi.org/10.3991/ijim.v14i01.11538

[7] L. Gupta, R. Jain and G. Vaszkun (2016) Survey of Important Issues in UAV Communication Networks. In IEEE Communications Surveys \& Tutorials, 18(2): pp. 1123-1152. https://doi.org/10.1109/comst.2015.2495297

[8] S. Singh, M. Woo, and C. Raghavendra (1998) Power-aware routing in Mobile Ad hoc Networks. In Proc. of the ACM/IEEE International Conference on Mobile Computing and Networking, pp. 181-190. IEEE. https://doi.org/10.1145/288235.288286

[9] C.-K. Toh, (2001) Maximum Battery Life Routing to Support Ubiquitous Mobile Computing in Wireless Ad hoc Networks. In IEEE Communications Magazine, 39(6): pp. 138-147. https://doi.org/10.1109/35.925682

[10] N.-C. Wang (2012) Power-aware Dual-tree-based Multicast Routing Protocol for Mobile Ad hoc Networks. In IET Communications, 6(7): pp. 724-732. https://doi.org/10. 1049/iet-com.2011.0073

[11] P. Zhao, X. Yang, W. Yu and X. Fu (2013) A Loose-Virtual-Clustering-Based Routing for Power Heterogeneous MANETs. in IEEE Trans. on Vehicular Tech., 62(5): pp. 2290-2302. https://doi.org/10.1109/tvt.2012.2237556

[12] Shiva Shankar, Golla Varaprasad, Hosahalli Narayanagowda Suresh (2014) Importance of Ondemand Modified Power-aware Dynamic Source Routing Protocol in Mobile Ad-hoc Networks. in IET Microwaves, Antennas \& Propagation, 8(7): pp. 459-464. https://doi.org/10.104 9/iet-map.2013.0230

[13] Xiaoyan Wang, Jie Li (2015) Improving the Network Lifetime of MANETs through Cooperative MAC Protocol Design. in IEEE Transactions on Parallel and Distributed Systems, 26(4): pp. 1010-1020. https://doi.org/10.1109/tpds.2013.110

[14] Chih-Min Chao, Jang-Ping Sheu, Cheng-Ta Hu (2003) Energy-conserving grid routing protocol in mobile ad hoc networks. in Proc. of Int. Conf. on Parallel Processing, pp. 265-272. https://doi.org/10.1109/icpp.2003.1240589

[15] Zheng-Yu Wu, Han-Tao Song (2010) Ant-based Energy-aware Disjoint Multipath Routing Algorithm for MANETs. In The Computer Journal, 53(2): pp. 166-176. https://doi.org/10.1093/comjnl/bxn007

[16] F. De Rango, F. Guerriero and P. Fazio (2012) Link-Stability and Energy-aware Routing Protocol in Distributed Wireless Networks. in IEEE Transactions on Parallel and Distributed Systems, 23(4): pp. 713-726. https://doi.org/10.1109/tpds.2010.160

[17] D. Zhang, Z. Yang et al. (2013) An Energy-Efficient Routing Protocol Using Movement Trends in Vehicular Ad hoc Networks. in The Computer Journal, 56(8): pp. 938-946. https://doi.org/10.1093/comjnl/bxt028

[18] Aqeel Taha et al. (2017) Energy Efficient Multipath Routing Protocol for Mobile Ad-Hoc Network Using the Fitness Function. in IEEE Access, 5: pp. 10369-10381. https://doi.org/10.11 09/access.2017.2707537

[19] R. A. Rehman, S. H. Ahmed and B. Kim (2017) OEFS: On-Demand Energy-Based Forwarding Strategy for Named Data Wireless Ad Hoc Networks. in IEEE Access, 5: pp. 6075-6086. https://doi.org/10.1109/access.2017.2684912

[20] J. Bai, Y. Sun, C. Phillips and Y. Cao (2018) Toward Constructive Relay-Based Cooperative Routing in MANETs. in IEEE Systems Journal, 12(2): pp. 1743-1754. https://doi.org/10.110 9/jsyst.2017.2721543

[21] I. Kacem, B. Sait, S. Mekhilef and N. Sabeur (2018) A New Routing Approach for Mobile Ad Hoc Systems Based on Fuzzy Petri Nets and Ant System. in IEEE Access, 6: pp. 65705-65720. https://doi.org/10.1109/access.2018.2878145 
[22] W. A. Jabbar, W. K. Saad and M. Ismail (2018) MEQSA-OLSRv2: A Multicriteria-Based Hybrid Multipath Protocol for Energy-Efficient and QoS-Aware Data Routing in MANET-WSN Convergence Scenarios of IoT. in IEEE Access, 6: pp. 76546-76572. https://doi.org/10.1109/access.2018.2882853

[23] H. Kanagasundaram and A. Kathirvel (2019) EIMO-ESOLSR: Energy Efficient and Securitybased Model for OLSR Routing Protocol in Mobile Ad-hoc Network. IET Communications, 13(5): pp. 553-559. https://doi.org/10.1049/iet-com.2018.5564

[24] RFC3561. [Online]. Available: https://www.ietf.org

[25] RFC4728. [Online]. Available: https://www.ietf.org

[26] Quy V.K., Ban N.T., Han N.D. (2018) A Multi-metric Routing Protocol to Improve the Achievable Performance of Mobile Ad Hoc Networks. In Modern Approaches for Intelligent Information and Database Systems. Studies in Computational Intelligence, 769. Springer. https://doi.org/10.1007/978-3-319-76081-0 38

[27] Quy V.K., Ban N.T., Han N.D. (2019) A High-Performance Routing Protocol for Multimedia Applications in MANETs. in Journal of Communications, 14(4): pp. 267-274. https://doi.org/10.12720/jcm.14.4.267-274

[28] Quy V.K and Hung L.N. (2020) A Trade-off between Energy Efficiency and High-Performance in Routing for Mobile Ad hoc Networks. in Journal of Communications, 15(3): pp. 263-269. https://doi.org/10.12720/jcm.15.3.263-269

[29] Sami Abduljabbar Rashid et al. (2020) Prediction Based Efficient Multi-hop Clustering Approach with Adaptive Relay Node Selection for VANET. In Journal of Communications, 15(4): pp. 332-344. https://doi.org/10.12720/jcm.15.4.332-344

[30] Quy V.K., Hung L.N., Han N.D. (2019) CEPRM: A Cloud-assisted Energy-Saving and Performance-Improving Routing Mechanism for MANETs. in Journal of Communications, 14(2): pp. 267-274. https://doi.org/10.12720/jcm.14.12.1211-1217

\section{$7 \quad$ Authors}

Vu Khanh Quy was born in Hai Duong Province, Vietnam, in 1982. He received his B.Sc. degree from Hung Yen University of Technology and Education (UTEHY) in 2007 and his M.Sc. degree from Posts and Telecommunications Institute of Technology (PTIT), in 2012. Currently, he is a PhD student at Faculty of Telecommunications 1, PTIT. His research interests include Wireless Communications, Mobile Ad-hoc Computing and Next-Generation Networks. Email: quyvk@utehy.edu.vn

Vi Hoai Nam was born in Phu Tho Province, Viet Nam, in 1986. He received his B.Sc. degree from the Posts and Telecommunications Institute of Technology, in 2009 and received his M.S. degree from Ha Noi University of Technology, in 2013. Currently, he is a lecturer at UTEHY. His research interests include Wireless Sensor Networks, Next-Generation Networks. Email: vihoainam@gmail.com

Dao Manh Linh was born in Hung Yen Province, Vietnam, in 1988. He received his B.Sc. degree from UTEHY in 2013 and his M.Sc. degree from Posts and Telecommunications Institute of Technology (PTIT), in 2016. Currently, he is a researcher at UTEHY. His research interests include Wireless Communications, Mobile Ad-hoc Network and Next-Generation Networks. daolinh1289@gmail.com

Article submitted 2020-01-11. Resubmitted 2020-03-12. Final acceptance 2020-03-14. Final version published as submitted by the authors. 